\title{
BRAF Mutations Open Doors for N-Ethyl-N- Nitrosourea-Induced Gliomagenesis
}

\author{
Robert S. McNeill, ${ }^{*}$ David M. Irvin, ${ }^{\dagger}$ and C. Ryan Miller ${ }^{* \dagger \delta} \Phi$
}

From the Pathobiology and Translational Science Graduate Program, * the Curriculum in Genetics and Molecular Biology, ${ }^{\dagger}$ the Division of Neuropathology, Department of Pathology and Laboratory Medicine, the Lineberger Comprehensive Cancer Center, ${ }^{\S}$ and the Department of Neurology and Neurosciences Center, "University of North Carolina School of Medicine, Chapel Hill, North Carolina

In this issue of the American Journal of Pathology, Wang et al $^{1}$ identified recurrent Braf mutations in N-ethyl-Nnitrosourea (ENU)-induced rat gliomas by DNA sequencing. Their results provide a platform for preclinical development of novel targeted therapies for $B R A F$-mutant gliomas. Precision oncology promises to revolutionize cancer therapy by stratifying tumors on the basis of their molecular characteristics and using rationally designed treatments in molecularly defined patient populations. ${ }^{2}$ Although the field of oncology is transitioning into the precision medicine era, conventional diagnostics, based on tissue type, tumor pathology, and patient demographics, remain essential to quality care. One natural pathological division for gliomas, the most common primary brain tumors in humans, is based on their invasion, with nondiffuse gliomas forming well-circumscribed tumors and diffuse gliomas invading the normal brain. ${ }^{3}$ Within these two broad diagnostic categories, gliomas are further subdivided on the basis of patient demographics into pediatric and adult diseases and further into specific diagnostic entities based on their histological appearance.

\section{Human Gliomas Are Genomically Heterogeneous}

Genomic analyses are necessary to clinically implement the concepts of precision medicine. Comprehensive genomic studies have revealed the genetic diversity of specific glioma entities and increased precision in defining relevant disease subtypes. For instance, integration of multiple genomic and molecular analyses determined that there are three molecular classes of adult lower-grade gliomas (grade II and III astrocytomas, oligoastrocytomas, and oligodendrogliomas, based on the World Health Organization 2007 classification scheme) that are more accurately represented by genetic testing for IDHI/2, ATRX, and TP53 mutations and chromosome $1 \mathrm{p}$ and $19 \mathrm{q}$ losses than by histological class. ${ }^{3,4}$ Large-scale genomic sequencing has also shown that pediatric gliomas are genomically distinct diseases compared to their histologically similar adult counterparts. ${ }^{5}$ Moreover, genomic analyses have shown that even within a single demographic group or histological entity, multiple molecular subtypes of disease exist. ${ }^{6,7}$ Our increased understanding of the molecular characteristics underpinning glioma pathogenesis and increased precision in defining disease subsets will hopefully lead to the development of treatments that target the molecular aberrations driving their genesis. However, to fully achieve the promise of precision medicine, it will be necessary to use preclinical models that match specific disease subsets not only in terms of pathology and demographics, but on genetic mechanisms as well.

R.S.M. is a Robert H. Wagner Scholar in the Pathobiology and Translational Science Graduate Program and was supported by the University of North Carolina (UNC)/Howard Hughes Medical Institute (HHMI) Graduate Training Program in Translational Medicine. D.M.I. was supported by the UNC/HHMI Graduate Training Program in Translational Medicine and training grants T32GM007092 and T32CA071341 from the National Institutes of Health. C.R.M. was a Damon Runyon-Genentech Clinical Investigator supported in part by a Clinical Investigator Award from the Damon Runyon Cancer Research Foundation (CI-45-09) and the UNC University Cancer Research Fund.

Accepted for publication July 17, 2016.

R.S.M. and D.M.I. contributed equally to this work.

Disclosures: None declared.

Address correspondence to C. Ryan Miller, M.D., Ph.D., University of North Carolina School of Medicine, 6109B Neurosciences Research Bldg, Campus Box 7250, Chapel Hill, NC 27599-7250. E-mail: rmiller@med. unc.edu 


\section{Modeling Gliomas Using ENU}

The first animal models of human gliomas were developed by treating either mice or rats with chemical carcinogens. ${ }^{8}$ One such carcinogen is ENU, a DNA ethylating agent that induces widespread DNA damage, resulting in singlenucleotide mutations, most commonly $\mathrm{T}: \mathrm{A}$ to $\mathrm{A}: \mathrm{T}$ transversions and T:A to $\mathrm{C}: \mathrm{G}$ transitions. ${ }^{9}$ Rats in particular have been treated with ENU either during embryonic development or postnatally to induce gliomagenesis, and the resulting gliomas were either characterized within the intact brain or harvested to establish adherent cell lines cultured in vitro in serum-containing media.

Serum-cultured cell lines established from either chemically induced rodent gliomas or naturally occurring human tumors (established cell lines) have been the backbone of preclinical glioma research since the late 1960s. ${ }^{8}$ These models were widely disseminated and routinely used, but their genetic make-up was largely unknown until the advent of large-scale genomic analyses, such as microarray profiling and next-generation sequencing. These techniques have shown that established cell lines harbor more extensive genomic abnormalities than human gliomas and do not faithfully recapitulate the molecular profiles of patient samples. $^{10,11}$ Reasons for these discrepancies include adaptation to nutrient-rich media and clonal selection. ${ }^{8}$ Nevertheless, gliomas that arise spontaneously within an intact rat brain after ENU treatment are not subject to the selection pressures of serum culture. Therefore, these models may more faithfully recapitulate the genomics of acutely isolated patient tumors. Whether mutations in certain genes are highly penetrant or whether multiple mutations converge on specific, common biological pathway(s) in ENU-induced gliomas remained unclear.

\section{ENU-Induced Rat Gliomas Harbor Mutant Braf}

The study by Wang et $\mathrm{al}^{1}$ determined the mutational profile of ENU-induced rat gliomas. ${ }^{1}$ Whole genome sequencing was performed on tumors from three BDIV and two BDIX rats induced with ENU at post-natal day 1. Somatic mutations ranged from 6354 to 13,807 per glioma, with a mean of 10,685 , but recurrent copy number alterations were absent in the tumors examined. Consistent with ENU-induced mutagenesis, the most common singlenucleotide mutations were $\mathrm{T}: \mathrm{A}$ to $\mathrm{A}: \mathrm{T}$ transversions and T:A to $C: G$ transitions. Between 35 and 93 of these somatic mutations were shown to be functional, causing amino acid changes (92\%), introduction of stop codons $(6 \%)$, or destruction of splice sites $(2 \%)$. Somatic mutations in Srrm2, Olr158, Il12rb, Map1b, Runx2, Rsrc2, and $T c f 21$ occurred in two of the five gliomas analyzed. The only mutation unique to all five was an $\mathrm{A}$ to $\mathrm{T}$ missense mutation corresponding to Braf $f^{V 545 E}$. Wang et $\mathrm{al}^{1}$ found that this mutation was analogous to the $B R A F^{V 600 E}$ mutation at both the nucleotide and amino acid levels in humans and mice. To confirm the occurrence of the $B r a f^{V 545 E}$ in ENU-induced rat gliomas, the authors expanded their cohort to another 33 BDIV and 12 BDIX gliomas. Sanger sequencing determined that all 45 also harbored the Braf $f^{V 545 E}$ mutation. Moreover, Braf $f^{V 545 E}$ mutant rat gliomas were immunoreactive to a human BRAF $^{\mathrm{V} 600 \mathrm{E}}$ antibody. Thus, they found that $\mathrm{Braf}^{V 545 E}$ is a ubiquitous mutation in ENU-induced gliomas in rats.

\section{Role of $B R A F$ in Tumorigenesis}

$B R A F$ encodes a serine/threonine protein kinase that activates the mitogen activated protein kinase effector arm of receptor tyrosine kinase signaling. Activating $B R A F$ mutations, particularly the most common V600E mutation, have been implicated in tumorigenesis in a variety of solid cancers, including melanoma (approximately 70\%), papillary thyroid cancer $(45 \%)$, and colorectal cancers (approximately 10\%). This mutation lies within the kinase domain and leads to constitutive activation of the protein and hyperactivation of mitogen activated protein kinase signaling. ${ }^{12}$

Genomic analyses have shown that $B R A F$ mutations occur in nondiffuse human gliomas, including pilocytic astrocytomas, gangliogliomas, and pleomorphic xanthoastrocytoma. ${ }^{13}$ More important, all three tumor types develop most commonly in children and young adults and may display the cytological features of their diffuse oligodendroglioma counterparts, including perinuclear halos, crisp nuclear membranes, and prominent nucleoli. However, unlike diffuse oligodendrogliomas, each of these tumors are slow growing, benign (World Health Organization grade I, pilocytic astrocytomas and gangliogliomas) to mildly aggressive (World Health Organization grade II, pleomorphic xanthoastrocytoma), and have discrete tumor margins. They are thus generally surgically curable. Nevertheless, when location precludes complete resection, adjuvant therapies are required. Drugs targeting $B R A F^{V 600 E}$ (BRAFi) have been approved for treatment of metastatic melanoma. ${ }^{14}$ However, resistance inevitably occurs through a variety of mechanisms, including reactivation of mitogen activated protein kinase signaling or alternate activation of parallel pathways. Thus, rationally designed combination therapies that target multiple kinases within the same pathway and/or alternate pathways may be necessary to circumvent BRAFi resistance. Moreover, BRAFi have shown mixed results in the treatment of $B R A F$-mutant gliomas. ${ }^{15}$ Case reports suggest that $B R A F^{V 600 E}$-mutant gliomas are sensitive to BRAFi, but this observation has not been confirmed in clinical trials. Developing more accurate preclinical models to determine the underlying biology of $B R A F$-mutant gliomas is necessary for efficient development of novel treatments. 
Historically, the histological and cytological features of ENU-induced rat gliomas, including the extent of brain invasion, have been variable, with most being diagnosed as oligodendrogliomas, astrocytomas, or mixed gliomas. ${ }^{16}$ The ENU-induced rat gliomas from the study by Wang et $\mathrm{al}^{1}$ showed cytological features similar to low-grade or anaplastic oligodendrogliomas, but lacked diffuse brain invasion. ${ }^{1}$ Taken together, the histopathology of these model tumors is consistent with the nondiffuse gliomas, such as pilocytic astrocytomas, gangliogliomas, or pleomorphic xanthoastrocytoma, and their lack of invasion further supports the use of this model for studying these entities. Indeed, a significant strength of this ENU-induced rat glioma model is the developmental timing of tumorigenesis that may mimic pediatric patients. ${ }^{1}$ ENU injections were performed in perinatal mice, mirroring the age at which $B R A F^{V 600 E}$ gliomas might be initiated in humans. This timing, plus the ubiquitous penetrance of Braf ${ }^{V 545 E}$ mutations, suggests that this preclinical model may prove beneficial in studying the basic biology of $B R A F$-mutant pediatric gliomas and developing targeted therapies.

\section{Using BRAF-Mutant Murine Models to Study Gliomagenesis and Develop Targeted Therapies}

The complete penetrance of $B R A F^{V 545 E}$ mutations in the ENU-induced rat gliomas described by Wang et $\mathrm{al}^{1}$ suggests that this mutation drives tumorigenesis. However, the study was underpowered to identify other significantly mutated genes that may cooperate with mutant $B R A F .{ }^{1}$ Future studies using whole exome sequencing, sophisticated statistical tools, and increased samples sizes could confirm the frequency of the $B R A F^{V 545 E}$ mutation and identify potential cooperating mutations, such as CDKN2A (Ink4aArf) loss, that frequently co-occur in human gliomas. ${ }^{17-19}$

In this regard, the role of $B R A F$ mutations in gliomagenesis has been explored experimentally. Overexpression of both wild-type $B R A F$ and $B R A F^{V 600 E}$ in immortalized astrocytes induces senescence in vitro. ${ }^{20}$ One study using genetically engineered mice found that $B R A F^{V 600 E}$ alone was not sufficient to induce gliomas, but cooperated with $C d k n 2 a$ deletion to do so. ${ }^{21}$ Moreover, gliomas from these genetically engineered mice were well demarcated and noninfiltrative. In addition, the $\mathrm{BRAF}^{\mathrm{V} 600 \mathrm{E}}$ kinase domain alone was sufficient to generate pilocytic astrocytoma when expressed in situ. ${ }^{22}$ Both of these studies examined the role of $B R A F^{V 600 E}$ in transformation of neonatal mouse neural stem cells. However, the role of $B R A F^{V 600 E}$ in gliomagenesis could differ on the basis of the cell of origin. The cellular origins of Braf-mutant, ENU-induced rat gliomas described in Wang et $\mathrm{al}^{1}$ remain unknown. Future studies should focus on elucidating the influence of cellular origin and cooperating mutations in $B R A F$-driven gliomagenesis.

Genetically faithful, $B R A F$-mutant preclinical glioma models will aid in the development of novel, targeted treatments. Preclinical data suggest that $B R A F$ is a viable target in $B R A F$-mutant gliomas. Indeed, genetic ablation of $B R A F$ with shRNA reduces growth of $B R A F^{V 600 E}$-mutant glioma cell lines. ${ }^{23}$ This suggests that $B R A F$ mutations are involved in tumor maintenance as well as initiation. The role of $B R A F^{V 600 E}$ in tumor maintenance has also been investigated pharmacologically. $B R A F^{V 600 E}$-mutant, patientderived xenografts and established cell lines are sensitive to the BRAFi PLX4720 in vitro. ${ }^{23,24}$ Moreover, intracranial genetically engineered mouse-derived allografts developed using Braf $^{V 600 E}$ driven, Ink4a-Arf null neural progenitor cells were also sensitive to PLX4720. ${ }^{18}$ These results implicate oncogene addiction to $B R A F^{\mathrm{V} 600 \mathrm{E}}$ in gliomas, suggesting that the rat gliomas described by Wang et $\mathrm{al}^{1}$ may be addicted to BRAF mutations as well. ${ }^{1}$

Despite the promise of single agent-targeted therapies, drug resistance has limited their therapeutic benefits in melanoma patients. Given the resistance patterns seen clinically, it is unlikely that BRAF-targeted therapies will be effective as single agents in gliomas. ${ }^{14}$ Therefore, the generation of rationally designed combination treatments is paramount. To this end, the use of genetically accurate preclinical model systems is necessary. Indeed, the BRAFi, PLX4720, has been tested in vivo in combination with radiation in $B R A F^{V 600 E}$ mutant patient-derived xenografts and in combination with the cyclin-dependent kinase $4 / 6$ inhibitor PD0332991 in BRAF V600E mutant, Ink4a/Arf deleted genetically engineered mouse-derived allografts. ${ }^{18,24}$ These preclinical studies showed that combination treatment improved survival compared to untreated and PLX4720-treated mice. Nevertheless, all mice eventually succumbed to disease. Thus, more preclinical studies using experimentally tractable models are required to identify effective, rationally designed combination therapies. Should ENU-induced gliomas prove to have the same cooperating mutations as $B R A F$-mutant human gliomas, use of tumor cells harvested from this model for orthotopic implantation in a manner similar to genetically engineered mouse-derived allografts may prove useful. ${ }^{1}$ Adapting the ENU model to culture and allografts will enable control of critical variables for preclinical drug studies, such as tumor latency and penetrance. ${ }^{8}$ Moreover, leveraging a panel of $\mathrm{Braf}^{\mathrm{V} 545 \mathrm{E}}$-mutant, ENU-induced rat gliomas harboring a variety of cooperating mutations will be useful in further elucidating BRAFi resistance mechanisms in gliomas, determining how co-occurring mutations influence resistance, and evaluating novel combination therapies both in vitro and in vivo.

\section{Conclusions}

With the genomics revolution, development of models that faithfully recapitulate specific tumor subtypes have become increasingly important. The work of Wang et $\mathrm{al}^{1}$ thus opens the door to ENU-induced rat gliomas, firmly placing them 
as a viable model for BRAF-mutant human gliomas. ${ }^{1}$ These ENU-induced gliomas will provide a platform for improved preclinical modeling and drug development.

\section{References}

1. Wang Q, Satomi K, Oh JE, Hutter B, Brors B, Nicolle D, Liu H, Wolf S, Wiestler O, Kleihues P, Koelsch B, Kindler-Röhrborn A, Ohgaki H: Braf mutations initiate the development of rat gliomas induced by postnatal exposure to n-ethyl-n-nitrosourea. Am J Pathol 2016, 186:2569-2576

2. Mendelsohn J: Personalizing oncology: perspectives and prospects. J Clin Oncol 2013, 31:1904-1911

3. Louis DN, Perry A, Reifenberger G, von Deimling A, FigarellaBranger D, Cavenee WK, Ohgaki H, Wiestler OD, Kleihues P, Ellison DW: The 2016 World Health Organization classification of tumors of the central nervous system: a summary. Acta Neuropathol 2016, 131:803-820

4. Cancer Genome Atlas Research Network: Comprehensive, integrative genomic analysis of diffuse lower-grade gliomas. N Engl J Med 2015, 372:2481-2498

5. Paugh BS, Qu C, Jones C, Liu Z, Adamowicz-Brice M, Zhang J, Bax DA, Coyle B, Barrow J, Hargrave D, Lowe J, Gajjar A, Zhao W, Broniscer A, Ellison DW, Grundy RG, Baker SJ: Integrated molecular genetic profiling of pediatric high-grade gliomas reveals key differences with the adult disease. J Clin Oncol 2010, 28:3061-3068

6. Brennan CW, Verhaak RG, McKenna A, Campos B, Noushmehr H, Salama SR, et al: The somatic genomic landscape of glioblastoma. Cell 2013, 155:462-477

7. Saratsis AM, Kambhampati M, Snyder K, Yadavilli S, Devaney JM, Harmon B, Hall J, Raabe EH, An P, Weingart M, Rood BR, Magge SN, MacDonald TJ, Packer RJ, Nazarian J: Comparative multidimensional molecular analyses of pediatric diffuse intrinsic pontine glioma reveals distinct molecular subtypes. Acta Neuropathol 2014, 127:881-895

8. McNeill RS, Vitucci M, Wu J, Miller CR: Contemporary murine models in preclinical astrocytoma drug development. Neuro Oncol $2015,17: 12-28$

9. Justice MJ, Noveroske JK, Weber JS, Zheng B, Bradley A: Mouse enu mutagenesis. Hum Mol Genet 1999, 8:1955-1963

10. Clark MJ, Homer N, O'Connor BD, Chen Z, Eskin A, Lee H, Merriman B, Nelson SF: U87mg decoded: the genomic sequence of a cytogenetically aberrant human cancer cell line. PLoS Genet 2010, 6: e1000832

11. Li A, Walling J, Kotliarov Y, Center A, Steed ME, Ahn SJ, Rosenblum M, Mikkelsen T, Zenklusen JC, Fine HA: Genomic changes and gene expression profiles reveal that established glioma cell lines are poorly representative of primary human gliomas. Mol Cancer Res 2008, 6:21-30

12. Cantwell-Dorris ER, O’Leary JJ, Sheils OM: Brafv600e: implications for carcinogenesis and molecular therapy. Mol Cancer Ther 2011, 10: 385-394
13. Horbinski C: To braf or not to braf: is that even a question anymore? J Neuropathol Exp Neurol 2013, 72:2-7

14. Bollag G, Tsai J, Zhang J, Zhang C, Ibrahim P, Nolop K, Hirth P: Vemurafenib: the first drug approved for braf-mutant cancer. Nat Rev Drug Discov 2012, 11:873-886

15. Collins VP, Jones DT, Giannini C: Pilocytic astrocytoma: pathology, molecular mechanisms and markers. Acta Neuropathol 2015, 129: $775-788$

16. Zook BC, Simmens SJ, Jones RV: Evaluation of enu-induced gliomas in rats: nomenclature, immunochemistry, and malignancy. Toxicol Pathol 2000, 28:193-201

17. Lawrence MS, Stojanov P, Polak P, Kryukov GV, Cibulskis K, Sivachenko A, et al: Mutational heterogeneity in cancer and the search for new cancer-associated genes. Nature 2013, 499:214-218

18. Huillard E, Hashizume R, Phillips JJ, Griveau A, Ihrie RA, Aoki Y, Nicolaides T, Perry A, Waldman T, McMahon M, Weiss WA, Petritsch C, James CD, Rowitch DH: Cooperative interactions of brafv600e kinase and cdkn2a locus deficiency in pediatric malignant astrocytoma as a basis for rational therapy. Proc Natl Acad Sci U S A 2012, 109:8710-8715

19. Schindler G, Capper D, Meyer J, Janzarik W, Omran H, HeroldMende C, Schmieder K, Wesseling P, Mawrin C, Hasselblatt M, Louis DN, Korshunov A, Pfister S, Hartmann C, Paulus W, Reifenberger G, von Deimling A: Analysis of braf v600e mutation in 1,320 nervous system tumors reveals high mutation frequencies in pleomorphic xanthoastrocytoma, ganglioglioma and extracerebellar pilocytic astrocytoma. Acta Neuropathol 2011, 121: 397-405

20. Jacob K, Quang-Khuong DA, Jones DT, Witt H, Lambert S, Albrecht S, Witt O, Vezina C, Shirinian M, Faury D, Garami M, Hauser P, Klekner A, Bognar L, Farmer JP, Montes JL, Atkinson J, Hawkins C, Korshunov A, Collins VP, Pfister SM, Tabori U, Jabado N: Genetic aberrations leading to mapk pathway activation mediate oncogene-induced senescence in sporadic pilocytic astrocytomas. Clin Cancer Res 2011, 17:4650-4660

21. Robinson JP, VanBrocklin MW, Guilbeault AR, Signorelli DL, Brandner S, Holmen SL: Activated braf induces gliomas in mice when combined with ink4a/arf loss or akt activation. Oncogene 2010, 29: $335-344$

22. Gronych J, Korshunov A, Bageritz J, Milde T, Jugold M, Hambardzumyan D, Remke M, Hartmann C, Witt H, Jones DT, Witt O, Heiland S, Bendszus M, Holland EC, Pfister S, Lichter P: An activated mutant braf kinase domain is sufficient to induce pilocytic astrocytoma in mice. J Clin Invest 2011, 121:1344-1348

23. Nicolaides TP, Li H, Solomon DA, Hariono S, Hashizume R, Barkovich K, Baker SJ, Paugh BS, Jones C, Forshew T, Hindley GF, Hodgson JG, Kim JS, Rowitch DH, Weiss WA, Waldman TA, James CD: Targeted therapy for brafv600e malignant astrocytoma. Clin Cancer Res 2011, 17:7595-7604

24. Dasgupta T, Olow AK, Yang X, Hashizume R, Nicolaides TP, Tom M, Aoki Y, Berger MS, Weiss WA, Stalpers LJ, Prados M, James CD, Mueller S, Haas-Kogan DA: Survival advantage combining a braf inhibitor and radiation in braf v600e-mutant glioma. J Neurooncol 2016, 126:385-393 